\title{
Treatment of pituitary prolactinoma reverses unresponsiveness to PDE5 inhibitor therapy in men with ED and SHPRL
}

\author{
Tao Jiang, Lei Zheng, Xiao-Ming Su, Jin-Qiang Peng, Dong-Cheng Sun, Quan-Lin Li, Zhi-Wei Zhang, \\ Fa-Peng Wang and Hui Jiang
}

Asian Journal of Andrology (2013) 15, 847-849; doi:10.1038/aja.2013.90; published online 29 July 2013

Dear Editor,

Four patients with erectile dysfunction (ED) induced by pituitary prolactinoma presented to our clinic from 2007 to 2008 . The patients had been misdiagnosed nearly 2 years earlier at other hospitals because severe ED was the initial symptom. None of these patients had experienced successful and satisfactory intercourse in approximately 2 years despite being treated with numerous medications, including the repeated administration of phosphodiesterase-5 inhibitors (PDE5is) (sildenafil $100 \mathrm{mg}$ and/or tadalafil $20 \mathrm{mg}$ ). However, after successful treatment for pituitary prolactinoma, the response to PDE5is improved dramatically. The four cases are described in this report.

Case 1: A 30-year-old male had a 2-year history of ED, with a lack of morning erections and sexual desire, as assessed using SIEDY Structured Interview question item 14- in the last three months, did you have more or less desire to make love'. His International Index of Erectile Function (IIEF-5) ${ }^{1}$ score was 5, and multiple administrations of sildenafil $100 \mathrm{mg}$ and tadalafil $20 \mathrm{mg}$ were ineffective (his IIEF-5 score had remained unchanged). He also experienced occasional dizziness and headaches. A physical examination (PE) revealed gynecomastia with lactation and normally developed external genitalia with a normal testicular size and texture. The results of the related hormone tests are presented in Table 1. Brain magnetic resonance imaging (MRI) revealed a $2.3 \times 2.9 \times 2.7 \mathrm{~cm}$ giant pituitary adenoma in the sellar region. Following tumour resection via the right nasal sinus under general anaesthesia, the pathology report indicated the presence of a pituitary adenoma. After 24 months of follow-up, the patient's prolactin (PRL) level was $600 \mathrm{IU} \mathrm{l}^{-1}$, and the gynecomastia, lactation and other symptoms disappeared. His IIEF-5 score at that time was 15 . Currently, sildenafil $100 \mathrm{mg}$ is effectively controlling his $\mathrm{ED}$, and he is able to achieve satisfactory sexual function (IIEF-5 score $>21$ ) (Table 2).

Case 2: A 47-year-old male had a 2-year history of ED with a lack of morning erections and sexual desire. His IIEF-5 score was 6 , and the repeated administration of sildenafil $100 \mathrm{mg}$ and tadalafil $20 \mathrm{mg}$ was ineffective (his IIEF-5 score increased to only 7). A PE revealed no gynecomastia or lactation and normal external genitalia with a normal testicular size and texture. The results of the related hormone tests are presented in Table 1. Brain MRI revealed a pituitary tumour $1.2 \mathrm{~cm}$ in diameter. After the transnasal resection of the tumour under general anaesthesia, the pathology report indicated the presence of a pituitary adenoma. After 24 months

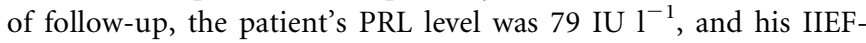
5 score was 19. Currently, the administration of sildenafil $100 \mathrm{mg}$ is effectively controlling his ED, and his IIEF-5 score has increased to 22 (Table 2).

Case 3: A 48-year-old male had a 2-year history of ED with a lack of morning erections and sexual desire. His IIEF-5 score was 6 , and multiple regimens of sildenafil $100 \mathrm{mg}$ and tadalafil $20 \mathrm{mg}$ were ineffective (his IIEF-5 score had remained unchanged). A PE revealed gynecomastia without lactation and normal external genitalia with a normal testicular size and texture. The results of the related hormone tests are presented in Table 1. Brain MRI revealed a pituitary tumour that was $1.0 \times$ $0.8 \times 0.7 \mathrm{~cm}$ in size. After the transnasal resection of the tumour under general anaesthesia, the pathology report indicated the presence of a pituitary adenoma. After 24 months of follow-up, the patient's PRL level was $768 \mathrm{IU} \mathrm{l}^{-1}$, and his IIEF-5 score was 15 . Currently, the administration of sildenafil $100 \mathrm{mg}$ is effectively controlling his ED (Table 2).

Case 4: A 35-year-old male had a 2-year history of ED without morning erections and a lack of sexual desire. He had been married for 1 year, but had not had successful sexual intercourse in nearly 2 years. The patient's IIEF-5 score was 5 , and multiple regimens of sildenafil $100 \mathrm{mg}$ and tadalafil $20 \mathrm{mg}$ were ineffective. A PE revealed no gynecomastia and normal external genitalia with a normal testicular size and texture. The results of the related hormone tests are presented in Table 1. Brain MRI revealed a pituitary tumour that was $0.9 \times 0.8 \times 0.7 \mathrm{~cm}$ in size. He was treated with bromocriptine because of the small tumour size. Two months after the administration of bromocriptine $5 \mathrm{mg}$, the patient's PRL level returned to normal, and he continued the use of bromocriptine at a dose of $2.5 \mathrm{mg}$ for 10 months. After 24 months of follow-up, the patient's PRL level is $300 \mathrm{IU} \mathrm{l}^{-1}$, and his IIEF-5 score is 22 (Table 2).

Additionally, none of these patients had a history of using medications known to affect PRL production, such as antihypertensives, antidepressants or neuroleptics. 
Table 1 Details of the cases and patient erectile function pre- and post-treatment for pituitary prolactinoma

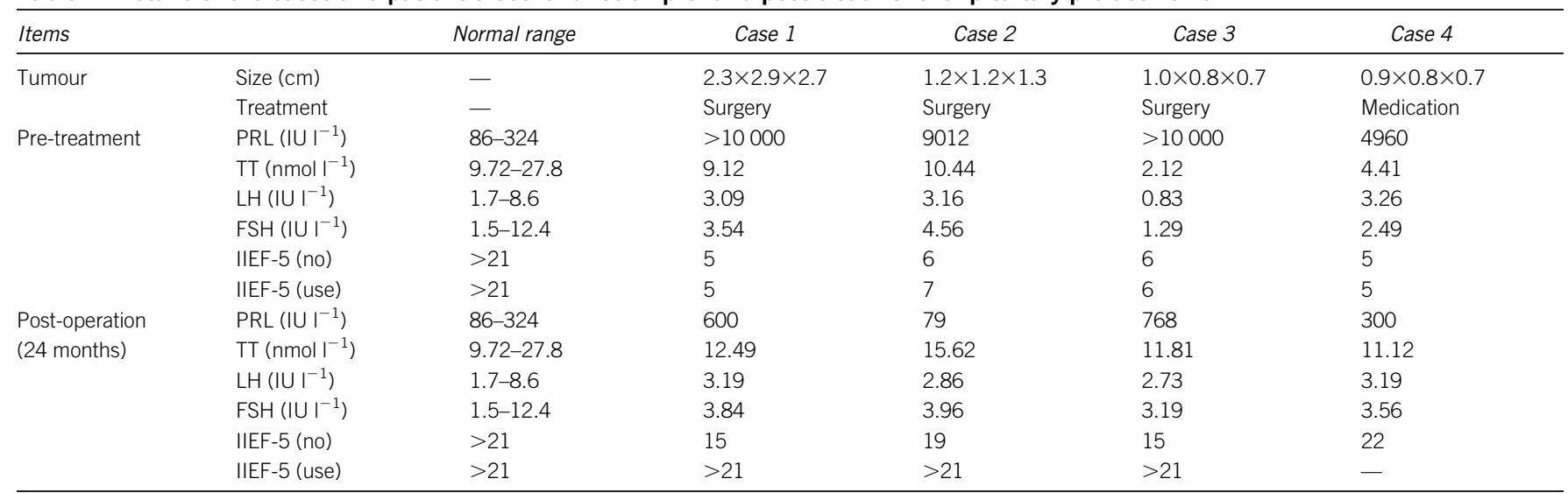

Abbreviations: FSH, follicle-stimulating hormone; IIEF-5 score: International Index of Erectile Function-5 score; IIEF-5 (no): IIEF-5 score without use of PDE5is; IIEF-5 (use): IIEF-5 score after the use of PDE5is; LH, luteinizing hormone; PRL, prolactinoma; TT, total testosterone.

Table 2 Patient erectile function pre- and post-treatment for pituitary prolactinoma

\begin{tabular}{lccccc}
\hline Cases & \multicolumn{2}{c}{ Pre-treatment } & & Post-treatment (12 months) \\
\cline { 2 - 3 } & IIEF-5 (no PDE5i) & IIEF-5 (with PDE5is) & & IIEF-5 (no PDE5i) & IEF-5 (with PDE5is) \\
\hline Normal range & $>21$ & 5 & 15 & $>21$ \\
Case 1 & 5 & 7 & 19 & $>21$ \\
Case 2 & 6 & 6 & 15 & $>21$ \\
Case 3 & 6 & 5 & 22 & - \\
Case 4 & 5 & 5 & \\
\hline
\end{tabular}

Abbreviations: IIEF-5: International Index of Erectile Function-5; PDE5i, phosphodiesterase-5 inhibitor.

PRL has a significant effect on male sexual function. However, severe hyperprolactinaemia (SHPRL, defined by a PRL level $>735$ $\mathrm{IU} \mathrm{l}^{-1}$ or $>35 \mathrm{ng} \mathrm{ml}^{-1}$ ) is relatively rare (less than $1 \%$ of men with ED). ${ }^{2}$ Pituitary tumours are one cause of SHPRL, which has a negative impact on sexual function.

Maggi et al. ${ }^{3}$ found that ED caused by a pituitary prolactinoma was associated with the induction of an androgen deficiency by HPRL. However, PRL-lowering drugs are capable of restoring both testosterone levels and libido, ${ }^{4}$ whereas testosterone replacement therapy is not as effective. ${ }^{5}$ This finding implies that the hypothalamus, not the Leydig cells, may be the major site affected by HPRL. The impairment of gonadal function at the level of the hypothalamus results in a subnormal basal secretion of the gonadotropins required for optimal testicular function. ${ }^{6}$

PDE5is are currently considered the first-line therapy for ED. However, in these cases, the repeated administration of PDE5is (sildenafil and tadalafil) for ED was ineffective. This lack of response, the impaired sexual desire and erectile function, and the decreased testosterone level may all have been caused by SHPRL. ${ }^{4,7}$ After the patients' PRL levels were corrected surgically or with medication, their sexual function improved (their IIEF-5 scores improved dramatically). These outcomes provide further support for the above hypotheses. Therefore, the optimal treatment of HPRL-related ED should be aimed at correcting the PRL level.

In this study, unresponsiveness to PDE5i therapy was reversed even if the PRL levels were not corrected to normal, although the exact reason is unclear. However, this may be linked to the increasing testosterone level. In cases 1 and 3 in this study, although the PRL levels did not normalize after surgery, testosterone increased to normal levels. Wang et al. ${ }^{8}$ analysed the sexual function of 70 patients with pituitary prolactinomas for a 6-month period following surgery and found that the degree of postoperative recovery of sexual function was closely associated with the postoperative testosterone level.

Although HPRL is an uncommon cause of ED, the measurement of serum PRL levels is recommended in cases with hypoactive sexual desire (or ED with a lack of sexual desire) and a documented biochemical testosterone deficiency because these symptoms may be associated with a serious and treatable prolactinoma. ${ }^{9}$ A study by Jabaloyas et al. ${ }^{10}$ supported the routine measurement of testosterone in the diagnosis and treatment of $\mathrm{ED}$, particularly in ED patients who did not respond to PDE5is, and promoted the measurement of PRL in ED patients with diminished testosterone levels.

In summary, although this study included a limited number of cases, it demonstrates that the treatment of pituitary prolactinoma can reverse unresponsiveness to PDE5i therapy in men with ED and SHPRL.

\section{AUTHOR CONTRIBUTIONS}

TJ, LZ, XMS and JQP cared for the patient and collected clinical information. DCS, ZWZ, FPW performed the laboratory tests. TJ, LZ drafted the manuscript, which was revised by HJ. QLL took part in critical discussion. 


\section{COMPETING FINANCIAL INTERESTS}

All authors declare that there are no competing financial interests.

1 Cappelleri JC, Rosen RC. The Sexual Health Inventory for Men (SHIM): a 5-year review of research and clinical experience. Int J Impot Res 2005; 17: 307-19.

2 Corona G, Mannucci E, Jannini EA, Lotti F, Ricca V et al. Hypoprolactinemia: a new clinical syndrome in patients with sexual dysfunction. J Sex Med 2009; 6: 1457-66.

3 Maggi M, Buvat J, Corona G, Guay A, Torres LO. Hormonal causes of male sexual dysfunctions and their management (hyperprolactinemia, thyroid disorders, $\mathrm{GH}$ disorders, and DHEA). J Sex Med 2013; 10: 661-77.
4 Corona G, Mannucci E, Fisher AD, Lotti F, Ricca V et al. Effect of hyperprolactinemia in male patients consulting for sexual dysfunction. J Sex Med 2007; 4: 1485-93.

5 Carter JN, Tyson JE, Tolis G, van Vliet S, Faiman C et al. Prolactin-screening tumours and hypogonadism in 22 men. N Engl J Med 1978; 299: 847-52.

6 Walia R, Bhansali A, Dutta P, Khandelwal N, Sialy R et al. Recovery pattern of hypothalamo-pituitary-testicular axis in patients with macroprolactinomas after treatment with cabergoline. Indian J Med Res 2011; 134: 314-9.

7 Bhasin S, Enzlin P, Coviello A, Basson R. Sexual dysfunction in men and women with endocrine disorders. Lancet 2007; 369: 597-611.

8 Wang T, LiSQ, HuangZ. Effects of transsphenoidal approach on thegonadal function of adult male prolactinoma patients (a report of 70 cases). Chin J Clin Neurosci2010; 18: 173-8.

9 Buvat J, Maggi M, Gooren L, Guay AT, Kaufman J et al. Endocrine aspects of male sexual dysfunctions. J Sex Med 2010; 7: 1627-56.

10 Jabaloyas JM. Hormonal etiology in erectile dysfunction. Arch Esp Urol. 2010; 63 621-7. 